\title{
Adult neural stem cell isolation from whole rat brain and neurosphere culture and differentiation
}

\author{
Ludwig PE ${ }^{1}$, Patil AA ${ }^{1,2}$ \\ Departments of ${ }^{1}$ Clinical and Translational Science, ${ }^{2}$ Neurosurgery, Creighton University School of Medicine, \\ Omaha, NE, USA. parkerludwig@creighton.edu
}

\begin{abstract}
Historically, it was thought that neurogenesis ceased by the end of development, but it is currently understood that neurogenesis continues throughout the life of an individual. This continued growth arises from neural stem or progenitor cells (NSCs) located in specific regions of the adult brain, including the subventricular zone and the dentate gyrus of the hippocampus. Increased understanding of the nature of these cells and their reaction to environmental stimuli is of paramount importance in the effort to discern their role and potential use in repair following neurological disruption. Neurosphere suspension culture is identified as an effective way of actualizing a self-renewing population of neural stem cells. This study demonstrated that adult rat neural stem cells could be effectively induced to differentiate into cells of astrocytic lineage through exposure to fetal bovine serum (FBS), and that the same population of precursor cells could be induced toward neuronal lineage through exposure to dibutyryl cyclic adenosine monophosphate (dcAMP). There were also observations noted regarding difficulty inducing cell attachment following enzymatic digestion of neurospheres, and potential effects on various types of assays, including migration assays (Fig. 7, Ref. 31). Text in PDF www.elis.sk. KEY WORDS: neural, stem cell, neurosphere, adult rat, cell culture, cell differentiation.
\end{abstract}

\section{Introduction}

Injuries and deficits of the nervous system have devastating effects on millions of people around the world. Estimates indicate that 500,000-700,000 children and 2.5 million adults experience traumatic brain injury each year (1-5). Annually, more than 795,000 people have strokes in the United States alone (6). Stroke remains a leading cause of death and functional decay in industrialized countries (7). The body of literature on repair in the central nervous system following injury focuses on neural stem or progenitor cells (NSCs). The purpose of this study was to explore the characteristics, proliferation, differentiation, and migration of neural stem cells derived from the whole brains of adult Sprague-Dawley rats, and to note difficulties encountered in matters of method and protocol to aid other investigators pursuing similar studies.

\section{Materials and methods}

\section{Media preparation}

The serum-free medium (SFM) used for neurosphere culturing was constituted of $1 \mathrm{ml}$ of B12 supplement (50x), $0.5 \mathrm{ml}$ of

${ }^{1}$ Department of Clinical and Translational Science, Creighton University School of Medicine, Omaha, NE, and ${ }^{2}$ Department of Neurosurgery, Creighton University School of Medicine, Omaha, NE, USA

Address for correspondence: Parker E Ludwig, Creighton University School of Medicine, 2500 California Plaza, Omaha, NE, USA 68178 Phone: +1.801 .635 .5374$

Acknowledgments: This work was supported by research funds provided by the Creighton University School of Medicine, Department of Surgery; and the 2017 Summer Research Program.
Glutamax (100x), $0.5 \mathrm{ml}$ of Gibco Antibiotic-Antimycotic (10,000 units/ml of penicillin, $10,000 \mu \mathrm{g} / \mathrm{ml}$ of streptomycin, and $25 \mu \mathrm{g} /$ $\mathrm{ml}$ of Amphotericin B), and $47.5 \mathrm{ml}$ of DMEM/F12 for every 50 $\mathrm{ml}$ of medium.

The washing medium consisted of $4 \mathrm{ml}$ of Gibco AntibioticAntimycotic as described above, and $46 \mathrm{ml}$ of high glucose DMEM for every $50 \mathrm{ml}$ of medium.

The 2x SFM was comprised of $2 \mathrm{ml}$ of B12 supplement (50x), $1 \mathrm{ml}$ of Glutamax (100x), $1 \mathrm{ml}$ of Gibco Antibiotic-Antimycotic, and $46 \mathrm{ml}$ of DMEM/F12 in $50 \mathrm{ml}$ of medium.

The astrocyte differentiation medium consisted of the SFM described above with the addition of $5 \%$ fetal bovine serum (FBS). The oligodendrocyte differentiation medium was comprised of the SFM with $30 \mathrm{ng} / \mathrm{ml}$ triiodo-L-thyronine (T3) supplement. The neuron differentiation medium was made up of the SFM with $1 \mathrm{mM}$ dibutyryl cyclic adenosine monophosphate (dcAMP).

All media were prepared using vacuum filtration through 0.2 $\mu \mathrm{m}$ polyethersulfone (PES) membrane rapid-flow filters.

Neural stem cell isolation from whole rat brain and neurosphere culturing

All procedures in this study were performed in accordance with the Guide for the Care and Use of Laboratory Animals outlined by the National Institutes of Health, and followed a process approved by the Institutional Animal Care and Use Committee (IACUC). Brain tissue was extracted from Sprague-Dawley rats, both males and females, within 30 minutes of their deaths. Fur was shaved, and the head and neck region was cleaned with $70 \%$ ethanol. The head was removed with sterile scissors and scalpels. Skin was removed from the superior portion of the head, and the 


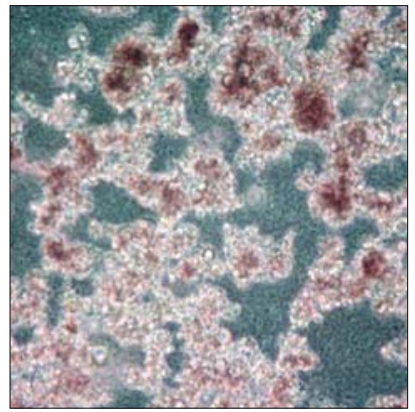

5 days

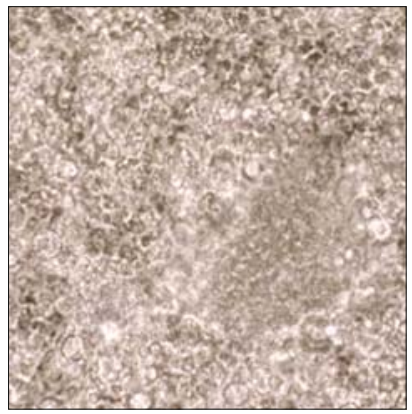

7 days

Fig. 1. Neurospheres in suspension culture 5 days and 7 days following isolation from brains of 8-week-old rats. Imaged at 40x magnification (with differences in exposure and color gradient).

skull was cracked with scissors. The overlying skull fragments were removed, and the brain was placed into $15 \mathrm{ml}$ of washing medium. The brain was then washed with fresh washing medium five times to minimize vascular debris. Brain tissue was minced manually and enzymatically in $0.1 \%$ collagenase for 45 minutes at $37{ }^{\circ} \mathrm{C}$. The resulting solution was centrifuged at $500 \mathrm{~g}$ for 5 minutes, and the supernatant was discarded. $5 \mathrm{ml}$ of SFM was added to the pellet, which was gently triturated. The resulting solution was strained through a $70 \mu \mathrm{m}$ tissue strainer; explant was discarded, and the strained solution was added to a T75 flask. An additional $5 \mathrm{ml}$ of medium was added to the flask in addition to $10 \mu \mathrm{L}$ of FGF2 (basic fibroblast growth factor) $(10 \mathrm{ng} / \mathrm{ml})$ and 10 $\mu \mathrm{L}$ of EGF (epidermal growth factor) $(10 \mathrm{ng} / \mathrm{ml})$. The flasks were placed in $37^{\circ} \mathrm{C}$ and left undisturbed for 3 days (Fig. 1).

$10 \mu \mathrm{L}$ quantities of EGF and FGF2 were added every three days due to the short half-life of FGF2. After 5-6 days, $5 \mathrm{ml}$ of culture medium (with cells) was transferred to a new T75 flask, and $5 \mathrm{ml}$ of 2x serum-free medium was added to each flask along with $10 \mu \mathrm{L}$ EGF and $10 \mu \mathrm{L}$ FGF2.

Cells were passaged using Accutase, when neurospheres reached approximately $150 \mu \mathrm{m}$ in diameter, which was typically after 6-10 days of culturing. Neurospheres were removed from flasks and centrifuged at $500 \mathrm{~g}$ for 5 minutes. The supernatant was removed, and each pellet was suspended in $1.5 \mathrm{ml}$ of Accutase for 30 minutes. The cells were spun again at $500 \mathrm{~g}$ for 5 minutes, and the supernatant was discarded. Cells were resuspended in 2 $\mathrm{ml} \mathrm{SFM}$, and $1 \mathrm{ml}$ was seeded into each T75 flask. $9 \mathrm{ml}$ of additional SFM was added to each flask in addition to $10 \mu \mathrm{L}$ EGF and $10 \mu \mathrm{L}$ FGF2.

\section{Immunofluorescence}

Characterization of the neurospheres was performed using immunofluorescence (IF). Cells from suspension culture were plated onto slides using a cytospin machine. The following primary antibodies raised in mouse were used: nestin (neuroectodermal stem cell marker), SOX-2 (sex determining region Y-box 2), GFAP (glial fibrillary acidic protein), Oct-3/4 (octamer binding transcription factor 3/4), Msi-1 (Musashi-1), NG2 (neural/glial antigen 2), and CD45 (cluster of differentiation 45). Primary an-
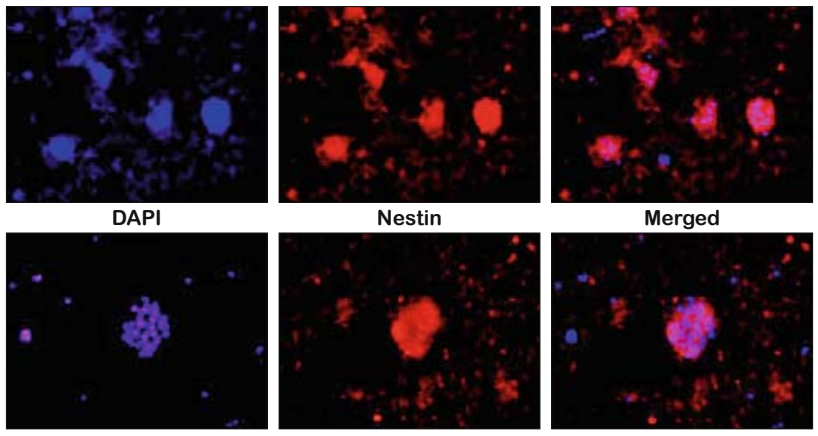

Merged
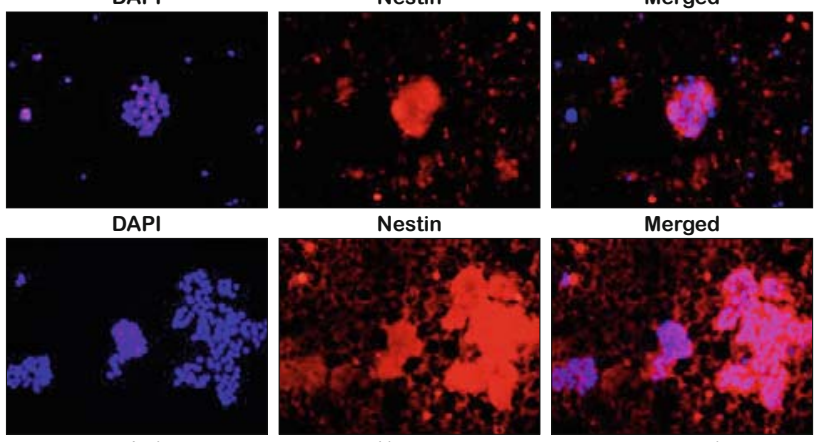

Merged
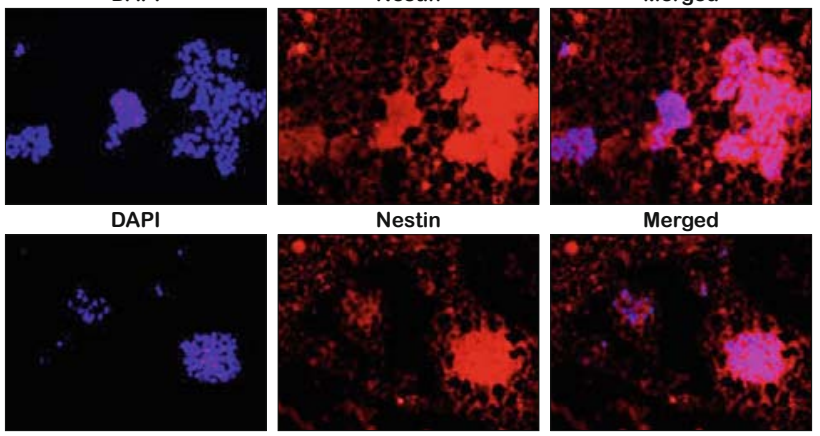

Merged
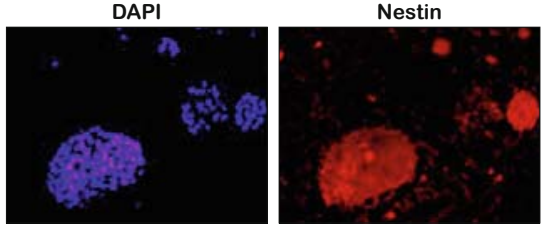

Nestin

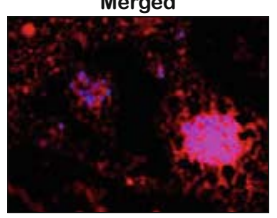

Merged
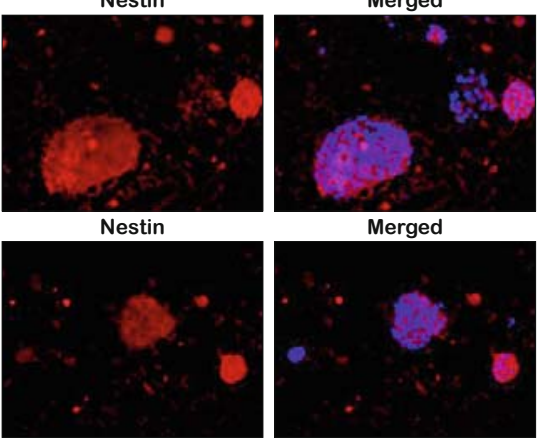

Nestin

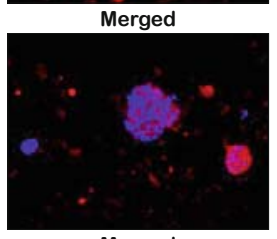

DAPI

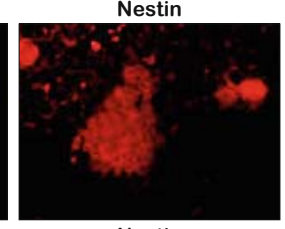

Nestin

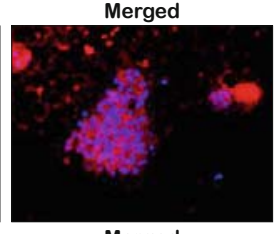

DAPI
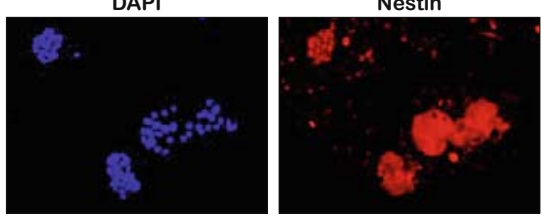

Nestin

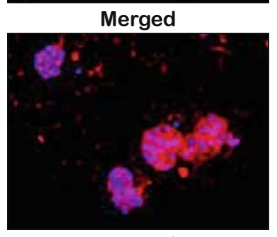

Merged

Fig. 2. Positive NSC markers. Neurosphere culture cells strongly associated with primary antibody against nestin with secondary antibody conjugated to $594 \mathrm{~nm}$ fluorochrome, primary antibody against SOX-2 with secondary antibody conjugated to $594 \mathrm{~nm}$ fluorochrome, primary antibody against Msi- 1 with secondary antibody conjugated to $594 \mathrm{~nm}$ fluorochrome, and primary antibody against GFAP with secondary antibody conjugated to $594 \mathrm{~nm}$ fluorochrome

tibodies raised in rabbit for NeuN (neuronal-specific nuclear protein), and Tuj1 (neuron-specific class III beta tubulin) were also used. The secondary antibodies used were donkey-anti-mouse 

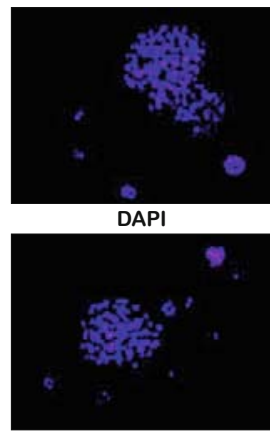

DAPI

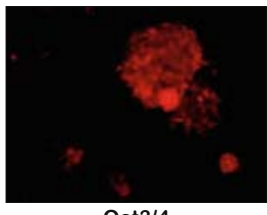

Oct3/4

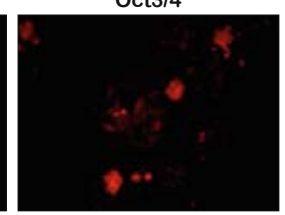

Oct3/4

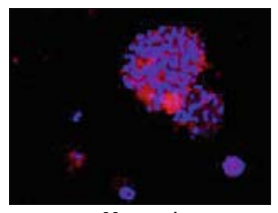

Merged

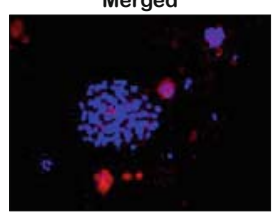

Merged

Fig. 3. Weakly-expressed OCT3/4 marker in images using primary antibody against OCT3/4 and secondary antibody conjugated to 594 nm fluorochrome.

with the fluorochrome at $594 \mathrm{~nm}$, and goat-anti-rabbit with 488 nm fluorochrome (Figs 2-6).

\section{Differentiation}

P3 neurospheres from culture were centrifuged and seeded into poly-L-ornithine- and laminin-coated chamber slides in their respective differentiation media as described above. In two cultures, neurospheres were enzymatically digested using Accumax for 15 minutes just prior to seeding in the chamber slides to observe the effect on cell attachment and differentiation. All cells, regardless of intended lineage, were cultured for 14 days to allow time for both attachment and differentiation. New media were added every two days. After differentiation had appeared to occur under phase contrast microscopy, cells were washed gently with SFM and fixed in $10 \%$ neutral buffered formalin solution for 30 minutes. The slides were then washed with PBS and underwent typical immunofluorescence (IF) protocol using the markers to be discussed later to identify the cell types (Figs 5-6).

\section{Transwell migration assay}

24-well plates with $8 \mu \mathrm{m}$ pore transwell inserts were used in the migration assays. The bottom wells were prepared with SFM and the appropriate concentrations of FGF2 and EGF in addition to human chorionic gonadotropin titrated to the following concentrations: $1 \mu \mathrm{g} / \mathrm{ml}, 0.5 \mu \mathrm{g} / \mathrm{ml}, 0.25 \mu \mathrm{g} / \mathrm{ml}, 0.125 \mu \mathrm{g} / \mathrm{ml}, 0.0625$ $\mu \mathrm{g} / \mathrm{ml}$, and $0 \mu \mathrm{g} / \mathrm{ml}$ (control). Total volume in the lower wells was $600 \mu \mathrm{L}$. To each laminin-coated insert was added $100 \mu \mathrm{L}$ of cell suspension, which was prepared by centrifuging a confluent T75 flask of neurospheres at $350 \mathrm{~g}$ for 5 minutes, removing the supernatant, resuspending pellet in Accutase for 30 minutes, centrifuging again at $350 \mathrm{~g}$ for 5 minutes, discarding the supernatant, and resuspending the pellet in $1.4 \mathrm{ml}$ of SFM (with FGF2 and EGF). Fourteen wells were utilized in each plate with two wells containing only the SFM and cell suspension, but no inserts. The migration assays ran for either 24 or 48 hours. Crystal violet and methylene blue were used in different assays to stain the cells to enable manual cell counting. In two assays each, uncoated inserts and poly-L-ornithine inserts were used following an otherwise identical protocol to observe any effect on cell attachment.
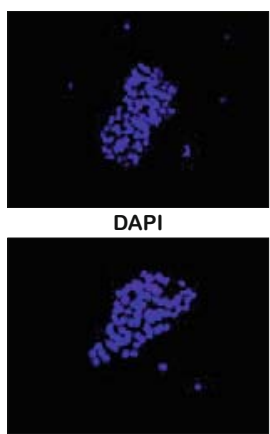

DAPI

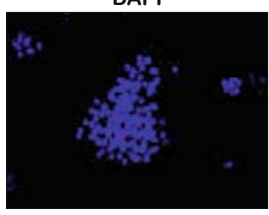

DAPI

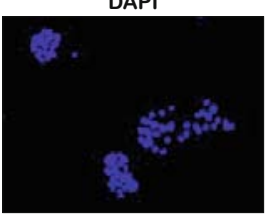

DAPI

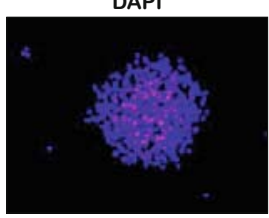

DAPI

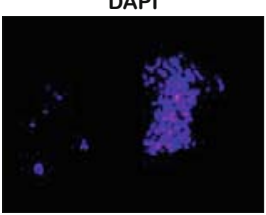

DAPI

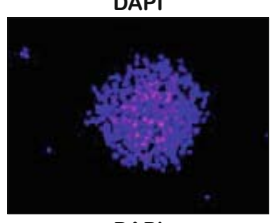

DAPI

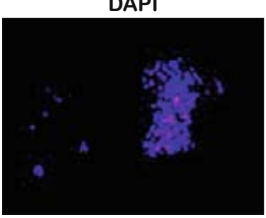

DAPI

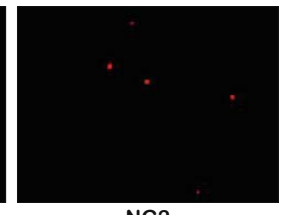

NG2

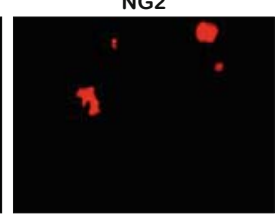

NG2

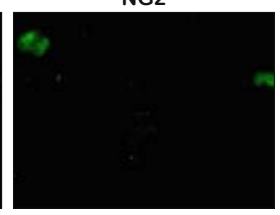

NeuN

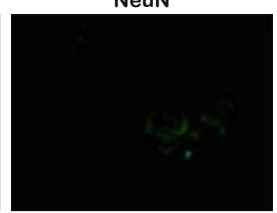

NeuN

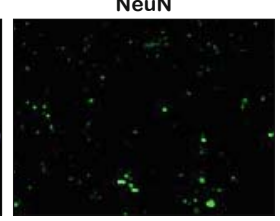

Tuj1

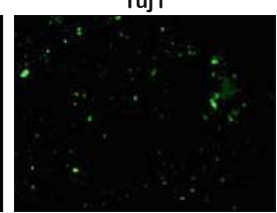

Tuj1

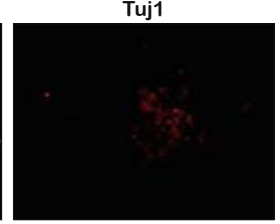

CD45

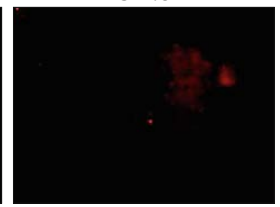

CD45

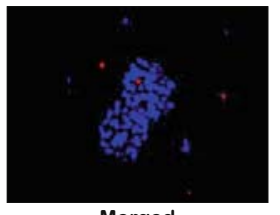

Merged

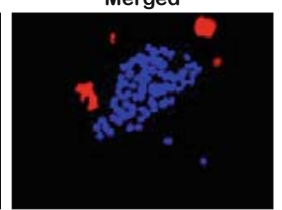

Merged

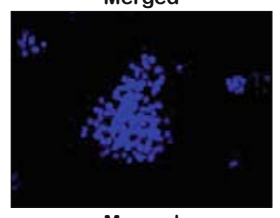

Merged

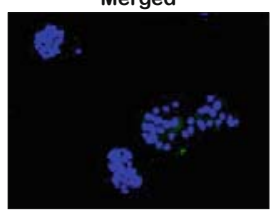

Merged

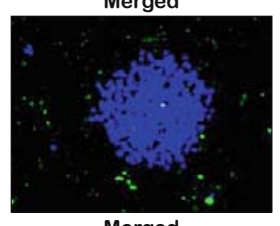

Merged

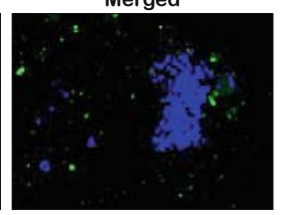

Merged

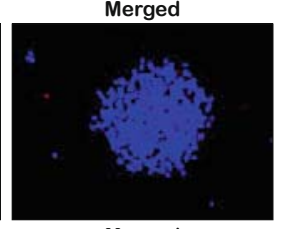

Merged

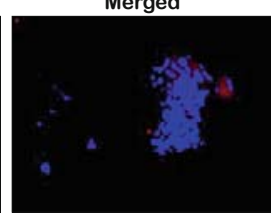

Merged

Fig. 4. Negative NSC markers. Antibodies targeting negative markers for neurosphere culture cells included primary antibody against NG2 with secondary antibody conjugated to $594 \mathrm{~nm}$ fluorochrome, primary antibody against NeuN with secondary antibody conjugated to $488 \mathrm{~nm}$ fluorochrome, primary antibody against Tuj1 with secondary antibody conjugated to $488 \mathrm{~nm}$ fluorochrome, and primary antibody against CD45 with secondary antibody conjugated to $594 \mathrm{~nm}$ fluorochrome.

Uncoated inserts were used in studies performed with the intent of observing the migration of cells across the membrane into a freely-floating state in the lower well (Fig. 7). Images of the 

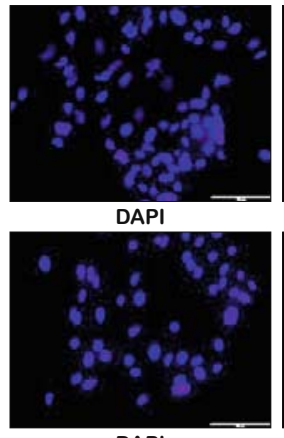

DAPI

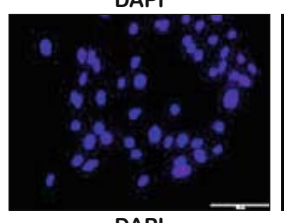

DAPI

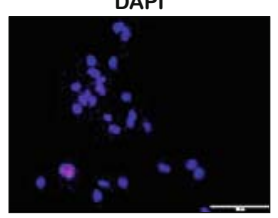

DAPI

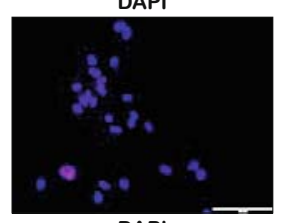

DAPI

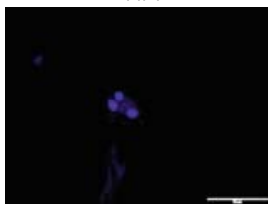

DAPI

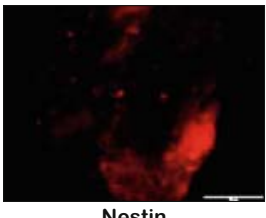

Nestin

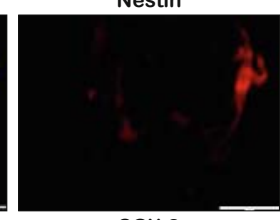

sox-2

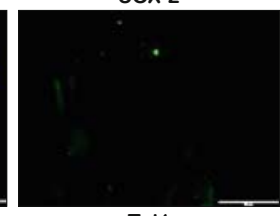

Tuj1

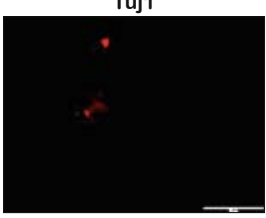

NG2

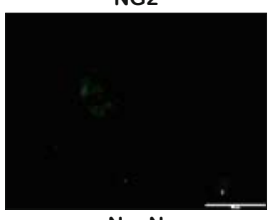

NeuN

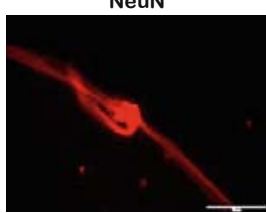

GFAP

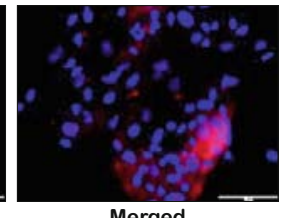

Merged

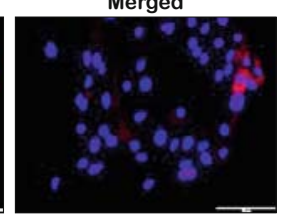

Merged

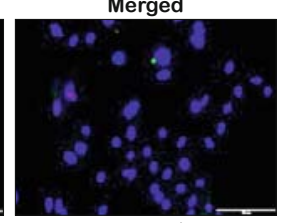

Merged

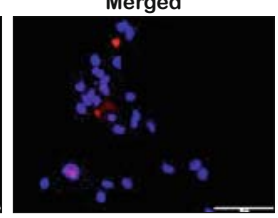

Merged

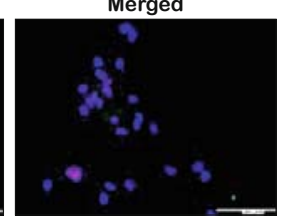

Merged

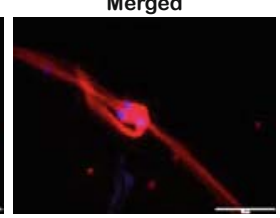

Merged

Fig. 5. Cells cultured in the astrocyte differentiation medium. GFAP was expressed by these cells, and could be seen in projections from the nuclei (with secondary antibody conjugated to $594 \mathrm{~nm}$ fluorochrome). All other markers were negative including primary antibodies against nestin, SOX-2, NG2, NeuN, andTuj1, with the secondary antibodies and conjugated fluorochromes as stated previously.

lower wells were taken at regular intervals, and viable cell quantity was quantified using MTT (3-(4,5-Dimethylthiazol-2-yl)-2,5Diphenyltetrazolium Bromide) assay.

\section{Migration quantification}

The inferior aspect of the coated membranes was stained using crystal violet. After removing the inserts, the superior side of the membrane was gently wiped with a cotton swab to remove cells. The remaining cells were fixed in $10 \%$ neutral buffered formalin solution for 5 minutes. This was followed by rinsing in PBS (phosphate buffered saline), then staining in crystal violet for 25 minutes. Brief destaining was performed using PBS, and the inserts were placed on microscope slides and imaged using a bottom-viewing microscope at 40x magnification with a 0.55
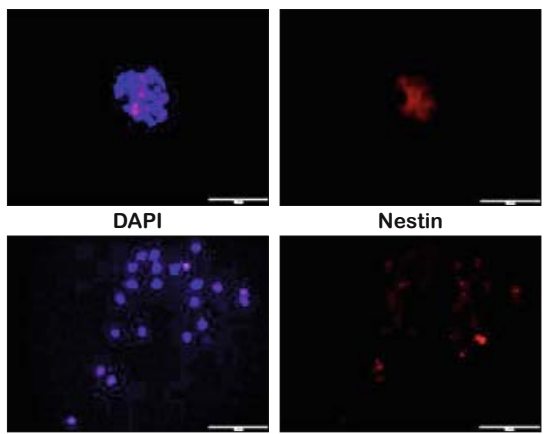

DAPI
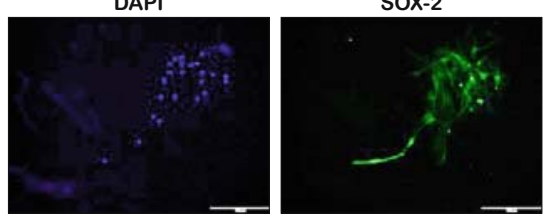

DAP

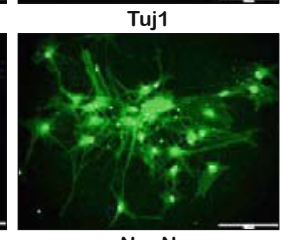

DAPI

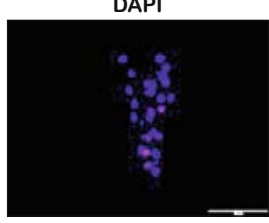

DAPI

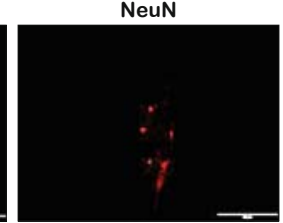

GFAP

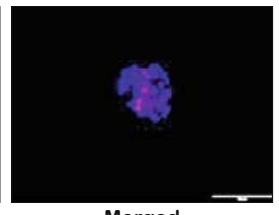

Merged

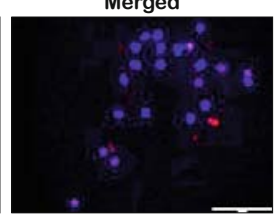

Merged

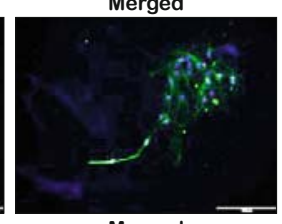

Merged

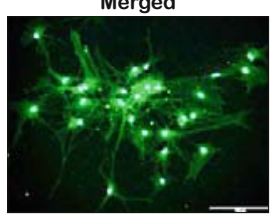

Merged

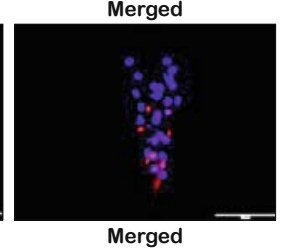

Fig. 6. Cells cultured in the neuronal differentiation medium. NeuN was strongly expressed by cells that had numerous, large projections around the nuclei; Tuj1 was expressed by some cells with limited projections from the nuclei (both with secondary antibodies conjugated to $488 \mathrm{~nm}$ fluorochrome). Other negative markers were not expressed; these were investigated using primary antibodies against nestin, SOX2, and GFAP, and secondary antibodies as described.

Bottom wells in migration assays

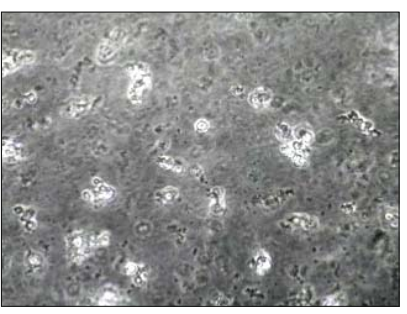

48 hours

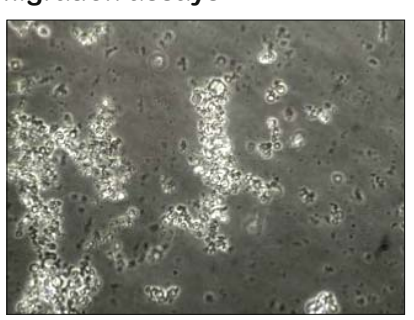

5 days
Fig. 7. Migration was noted across the transwell membrane as cells were visualized in bottom wells containing varying concentrations of HCG using phase contrast microscopy. Both images are taken from wells containing $1 \mu \mathrm{g} / \mathrm{ml} \mathrm{HCG}$ : on the left is an image taken 48 hours after seeding in the insert, and the right image is after 3 additional days of culture; note expansion of neurospheres

mm field of view. Efforts were made to count cells manually, and differences in means were to be evaluated first with ANOVA (analysis of variance) then with t-test between individual groups to determine statistically significant differences. 
The MTT assay was conducted using $5 \mathrm{mg} / \mathrm{ml}$ stock solution of MTT in phosphate buffered saline (PBS). The contents of each well were centrifuged at $5000 \mathrm{rpm}$ for 5 minutes, and the supernatant was discarded. Each pellet was resuspended in $100 \mu \mathrm{L}$ of the MTT stock solution and incubated at $37^{\circ} \mathrm{C}$ for 2 hours. $50 \mu \mathrm{L}$ of dimethyl sulfoxide (DMSO) was added to each tube, and after aspirating, the contents were centrifuged again at $5000 \mathrm{rpm}$ for 2 minutes to force any debris to the bottom. The supernatants were added to wells in a 96-well plate, and read at $560 \mathrm{~nm}$.

\section{Results}

\section{Culturing and differentiation}

Neurospheres comprised of neural stem/progenitor cells were successfully cultured as determined by their expression of nestin, SOX-2, Musashi-1, GFAP, and non-expression of CD45, NG2, NeuN, and Tuj1, and their ability to differentiate into both neuronal and glial lineages (Figs 2 and 4).

Nestin, SOX-2, and musashi-1 were strongly expressed by the neurospheres (Fig. 2). GFAP was also expressed quite consistently. Oct-4 was expressed by some cells, but did not present the strong fluorescence that the other positive markers did (Fig. 3). A review of literature leads one to expect some expression of oct-4 in adult stem cells, but potentially with lower expression than is typical in embryonic stem cells (8-10).

NG2, NeuN, and Tuj1 were chosen as markers of differentiation with NG2 being expressed by oligodendroglial precursors, NeuN expression by mature postmitotic neurons, and the expression of Tuj1 by immature neurons (11-13). An antibody for CD45 was used to rule-out a hematopoietic identity or origin in the cells being investigated (14). There was no observable expression of NG2, NeuN, Tuj1, or CD45 by the spheres of cells with an absence of any colocalization between antibody signals and those of DAPI (4',6-diamidino-2-phenylindole).

The cells cultured in the astrocyte differentiation medium expressed GFAP, and no longer expressed NSC markers, such as: nestin and SOX-2. It should also be noted that these cells did not express Tuj1, NG2, and NeuN (Fig. 5). The cells cultured in the oligodendrocyte differentiation medium failed to attach in either the attempts using complete neurospheres or those in which the spheres were broken up using Accumax prior to culturing, so no information regarding potential differentiation was obtained. The cells cultured in the neuron differentiation medium were visualized at varying levels of differentiation (Fig. 6). The cells noted to have subjectively more expansive projections strongly expressed NeuN, while some cells with smaller/fewer projections expressed Tuj1. These cells of neuronal lineage failed to express nestin, SOX-2, NG2, and GFAP.

Of the differentiation cultures with neurospheres digested by Accumax just prior to seeding, none resulted in attached cells that could be stained and visualized. The cultures were maintained for 25 days, with continued failure to attach throughout the period.

\section{Migration assays}

While multiple attempts were made to optimize the protocol for the use of the transwell plates in the migration assays, a suitable method of quantification was not realized. Efforts to stain cells on the underside of the transwell inserts after staining with crystal violet or methylene blue did not yield compelling results. Some objects that initially appeared to be cells were determined to be bubbles formed as the inserts were placed on slides following staining; these objects were absent, when the inserts were visualized in the transwell plates with the imaged surface in no contact with other surfaces. The lack of significant staining observed in these objects supported this designation.

There was no difference observed among the plates coated with laminin, those coated with poly-L-ornithine, and those that were left uncoated. There was lack of attachment noted regardless of the insert coating.

The MTT assays of the bottom well contents suggested a trend toward an increased migration with increasing HCG concentration, however, the results were not significant. This lack of significance could result from lack of a relationship between HCG administration and increased NSC migration, or it could be due to the small sample size as only two different migration cultures, each with two wells per concentration, were subjected to the MTT assay. The first MTT assay was conducted using a 2-hour incubation period, and the second assay was incubated overnight to increase the intensity of the spectrophotometric reading.

\section{Discussion}

NSCs can be difficult to culture effectively. The initial efforts in this project were to induce the cells to attach to poly-Lornithine- and laminin-coated flasks. There were no successful attempts to culture attached NSCs without inducing differentiation. This prompted a switch to suspension culture, which proved effective in enabling neurospheres to form, and cells to proliferate and maintain stem cell markers. Others have documented benefits and difficulties associated with the use of neurosphere culture, and the experience of the authors supports a suggestion that suspended neurosphere culture remains an optimal method for perpetuating NSC growth and proliferation without causing undesired differentiation (15-17). The use of neurosphere cultures has been expanded to the culture of cells from human neoplasms as well (18, 19). To prevent differentiation in the neurosphere culture, it was of importance that the medium remained free of serum, and that EGF and FGF2 were replaced regularly.

It is documented that dcAMP is an effective means of inducing NSC differentiation into neurons; however, previous studies illustrated this effect in embryonic cells, in other species such as: mice and humans, or with an emphasis on the administration of dcAMP in combination with other factors such as: interferon- $\gamma$ $(20,21)$. This project confirmed that dcAMP, as the sole signaling factor added to medium, is effective at inducing neuronal differentiation from adult rat neural stem cells as derived from the whole brains of rats. It was noted that cells of neuronal lineage could be visualized at varying stages of differentiation as was indicated by 
the expression of Tuj1 by cells with two projections of limited size, and the expression of NeuN by cells with more expansive networks of projections that were evidently more mature (Fig. 6).

FBS was used in the astrocyte differentiation medium due to the effectiveness of serum at inducing astrocytic differentiation according to the reports in literature (22-25). It was demonstrated that FBS is an effective signal for inducing astrocyte differentiation in progenitor cells derived from the whole brains of adult rats. The cells exposed to FBS in culture were identified as being of astrocyte lineage due to their expression of GFAP, and negativity for all other pertinent markers examined (Fig. 5).

The lack of attachment following enzymatic digestion of the neurospheres prior to seeding led to wondering about what disrupted this process. A search of literature did not immediately resolve the question regarding potential effects of Accumax on neural stem cell attachment and differentiation. Attachment was not achieved after 25 days in differentiation culture with any of the differentiation media described previously. It would not be unreasonable to wonder if the enzymatic cleavage has a longlasting effect on the attachment capabilities of adult neural stem cells, or if the proximity of cells inherent in neurosphere aggregation and potential paracrine or juxtacrine signaling is requisite for the attachment and/or differentiation of neural stem cells. Further investigation into these questions is needed before any conclusions can be drawn.

The results obtained from the migration studies do not validate conclusions regarding the effects of HCG on neural stem cell migration. The difficulties associated with these migration assays are mentioned to aid those intending to do similar studies in the future. Though migration did occur, as evidenced by the presence of neurospheres in the bottom wells, the migration was not quantifiable by counting cells attached to the inferior surface of the insert membrane due to lack of attachment. The neurospheres were treated with Accutase prior to being placed inside the transwell inserts to divide them into individual cells better able to pass through the 8 $\mu \mathrm{m}$ pores; it is unclear if this enzymatic activity inhibited the attachment abilities of the cells, as discussed regarding the use of Accumax before differentiation studies. A cell viability assay such as MTT may prove a more efficacious means of quantifying cells in migration assays using transwell plates. However, it should be noted that Wang et al (26) reported a successful quantification of glioblastoma multiforme (GBM) neurosphere cells using a similar method to that attempted in this study, but with the use of DAPI instead of crystal violet or methylene blue. There is also no mention of enzymatic digestion of the cells immediately prior to seeding in the GBM study. Others have reported success in the use of standard Boyden chamber technique (27-31). Comparison of the protocol employed in this study and migration assay protocols reported in literature reveals that enzymatic digestion just before seeding could be a likely factor impeding attainment of readily quantifiable results, but this would need to be verified through additional study. The effects of HCG on NSC migration warrant further investigation.

There is continued interest in the study of stem cells to ascertain methods of improving prognoses for devastating conditions of the human nervous system. While much has been explored in relation to neural stem cells, there remain significant information to be elucidated. Rats provide a good model for animal studies that could mimic many aspects of human disease, development, and cellular processes. Much of the published literature focuses on stem cells derived from embryos; while this emphasis has gleaned vast amounts of invaluable information, there is great potential benefit to be derived from more extensive study of cells from adults. There could be notable differences between neural stem cells of adults and embryos that still need to be identified. Further study of adult neural stem cells may also have important clinical implications as many of the disease processes affecting the general population, such as: stroke and traumatic injury, arise in adults. Further investigation into adult neural stem cell proliferation, differentiation, and migration is of paramount importance.

\section{References}

1. Centers for Disease Control and Prevention. Report to Congress on Traumatic Brain Injury in the United States: Epidemiology and Rehabilitation. National Center for Injury Prevention and Control, Division of Unintentional Injury Prevention, Atlanta, GA; 2015.

2. Doan N, Patel M, Doan H, Janich K. Traumatic Brain Injury. Int J Phys Med Rehabil (Internet) 2016 (cited 2017 Apr 13); 04. www.omicsonline.org/open-access/traumatic-brain-injury-2329-9096-1000e120. php?aid $=82968$.

3. Dewan MC, Mummareddy N, Wellons JC, Bonfield CM. Epidemiology of Global Pediatric Traumatic Brain Injury: Qualitative Review. World Neurosurg 2016; 91: 497-509.e1.

4. Wilde EA, Hunter Bilger ED. Pediatric traumatic brain injury: Neuroimaging and neurorehabilitation outcome. Neurorehabilitation 2012; $245-260$.

5. Li L, Liu J. The effect of pediatric traumatic brain injury on behavioral outcomes: a systematic review: Review. Dev Med Child Neurol 2013; 55: $37-45$.

6. CDC. Prevalence of Stroke - United States, 2006-2010 (Internet). (cited 2017 Apr 13). www.cdc.gov/mmwr/preview/mmwrhtml/mm6120a5. htm?s_cid=mm6120a5_w.

7. Taylor TN, Davis PH, Torner JC, Holmes J, Meyer JW, Jacobson MF. Lifetime Cost of Stroke in the United States. Stroke 1996; 27 : 1459-1466.

8. Tai MH, Chang CC, Olson LK, Trosko JE. Oct4 expression in adult human stem cells: evidence in support of the stem cell theory of carcinogenesis. Carcinogenesis 2005; 26: 495-502.

9. Verfaillie CM. Adult stem cells: assessing the case for pluripotency. Trends Cell Biol 2002; 12: 502-508.

10. Jiang Y, Jahagirdar BN, Reinhardt RL, Schwartz RE, Keene CD, Ortiz-Gonzalez XR et al. Pluripotency of mesenchymal stem cells derived from adult marrow. Nature 2002; 418: 41-49.

11. Karram K, Chatterjee N, Trotter J. NG2-expressing cells in the nervous system: role of the proteoglycan in migration and glial-neuron interaction. J Anat 2005; 207: 735-744.

12. Gusel'nikova VV, Korzhevskiy DE. NeuN As a Neuronal Nuclear Antigen and Neuron Differentiation Marker. Acta Naturae 2015; 7: 42-47. 
13. Menezes JR, Luskin MB. Expression of neuron-specific tubulin defines a novel population in the proliferative layers of the developing telencephalon. J Neurosci Off J Soc Neurosci 1994; 14: 5399-5416.

14. Nakano A, Harada T, Morikawa S, Kato Y. Expression of leukocyte common antigen (CD45) on various human leukemia/lymphoma cell lines. Acta Pathol Jpn 1990; 40: 107-115.

15. Jensen JB, Parmar M. Strengths and limitations of the neurosphere culture system. Mol Neurobiol 2006; 34: 153-161.

16. Mori H, Fujitani T, Kanemura Y, Kino-oka M, Taya M. Observational examination of aggregation and migration during early phase of neurosphere culture of mouse neural stem cells. J Biosci Bioeng 2007; 104: 231-234.

17. Ma BF, Liu XM, Xie XM, Zhang AX, Zhang JQ, Yu WH et al. Slower cycling of nestin-positive cells in neurosphere culture. NeuroReport 2006; 17: 377-381.

18. Guerrero-Cázares H, Chaichana KL, Quiñones-Hinojosa A. Neurosphere culture and human organotypic model to evaluate brain tumor stem cells. Methods Mol Biol Clifton NJ 2009; 568: 73-83.

19. Azari H, Millette S, Ansari S, Rahman M, Deleyrolle LP, Reynolds BA. Isolation and Expansion of Human Glioblastoma Multiforme Tumor Cells Using the Neurosphere Assay. J Vis Exp JoVE (Internet) 2011. www. ncbi.nlm.nih.gov/pmc/articles/PMC3227195/

20. Lee SH, Lumelsky N, Studer L, Auerbach JM, McKay RD. Efficient generation of midbrain and hindbrain neurons from mouse embryonic stem cells. Nat Biotechnol 2000; 18: 675-679.

21. Zahir T, Chen YF, MacDonald JF, Leipzig N, Tator CH, Shoichet MS. Neural stem/progenitor cells differentiate in vitro to neurons by the combined action of dibutyryl cAMP and interferon-gamma. Stem Cells Dev 2009; 18: 1423-1432.

22. Haas C, Neuhuber B, Yamagami T, Rao M, Fischer I. Phenotypic analysis of astrocytes derived from glial restricted precursors and their impact on axon regeneration. Exp Neurol 2012; 233: 717-732.
23. Brunet JF, Allaman I, Magistretti PJ, Pellerin L. Glycogen metabolism as a marker of astrocyte differentiation. J Cereb Blood Flow Metab Off J Int Soc Cereb Blood Flow Metab 2010; 30: 51-55.

24. Hu JG, Zhang YX, Qi Q, Wang R, Shen L, Zhang C et al. Expression of BMP-2 and BMP-4 proteins by type-1 and type- 2 astrocytes induced from neural stem cells under different differentiation conditions. Acta Neurobiol Exp (Warsz) 2012; 72: 95-101.

25. Fraichard A, Chassande O, Bilbaut G, Dehay C, Savatier P, Samarut $\mathbf{J}$. In vitro differentiation of embryonic stem cells into glial cells and functional neurons. J Cell Sci 1995; 108: 3181-3188.

26. Wang SD, Rath P, Lal B, Richard JP, Li Y, Goodwin CR et al. EphB2 receptor controls proliferation/migration dichotomy of glioblastoma by interacting with focal adhesion kinase. Oncogene 2012; 31: 5132-5143.

27. Imitola J, Raddassi K, Park KI, Mueller FJ, Nieto M, Teng YD et al. Directed migration of neural stem cells to sites of CNS injury by the stromal cell-derived factor $1 \alpha / \mathrm{CXC}$ chemokine receptor 4 pathway. Proc Natl Acad Sci 2004; 101: 18117-18122.

28. Sun L, Lee J, Fine HA. Neuronally expressed stem cell factor induces neural stem cell migration to areas of brain injury. J Clin Invest 2004; 113: 1364-1374.

29. Widera D, Holtkamp W, Entschladen F, Niggemann B, Zänker K, Kaltschmidt B et al. MCP-1 induces migration of adult neural stem cells. Eur J Cell Biol 2004; 83: 381-387.

30. Heese O, Disko A, Zirkel D, Westphal M, Lamszus K. Neural stem cell migration toward gliomas in vitro. NeuroOncol 2005; 7: 476-484.

31. Aarum J, Sandberg K, Haeberlein SLB, Persson MAA. Migration and differentiation of neural precursor cells can be directed by microglia. Proc Natl Acad Sci 2003; 100: 15983-15988.

Received September 25, 2018. Accepted October 29, 2018. 